\title{
Implication of Urban and Peri-Urban Agricultural Wastewater Irrigation to Malaria Transmission in Kumasi
}

\author{
Amina Abubakari ${ }^{1,2^{*}}$, John Asiedu Larbi ${ }^{1}$ and Robert Clement Abaidoo ${ }^{1}$ \\ ${ }^{1}$ Department of Theoretical and Applied Biology, Kwame Nkrumah University of Science and Technology, Kumasi-Ghana \\ ${ }^{2}$ Department of Laboratory Technology, Faculty of Health Sciences, Kumasi Technical University, Kumasi, Ghana
}

*Corresponding author: Amina Abubakari, Department of Theoretical and Applied Biology, Kwame Nkrumah University of Science and Technology, Kumasi, Ghana, Tel: +233-264-250-658; Fax: +233-3220-22387; E-mail: aminaabubakari@yahoo.com

Received date: July 19, 2018; Accepted date: August 14, 2018; Published date: August 22, 2018

Copyright: (c) 2018 Abubakari A et al. This is an open-access article distributed under the terms of the Creative Commons Attribution License; which permits unrestricted use; distribution; and reproduction in any medium; provided the original author and source are credited.

\begin{abstract}
Malaria transmission within areas of wastewater irrigation activities was the focus of this study where seven thousand and seventeen (7017) progressive data from health facilities within seventy-seven (77) communities were assessed. Results indicated approximately 3698 and 3319 cases of malaria, respectively for areas of wastewater irrigation activities and areas devoid of wastewater irrigation activities. Statistical result at $95 \%$ confidence interval (CI) gave a P-value of 0.1914 between these two multi-urban and peri-uran settings. The mean \pm SEM of $A$ (irrigation activity zones) was $13 \pm 3.816(\mathrm{~N}=4)$ and mean $\pm \mathrm{SEM}$ of $\mathrm{B}$ (non-irrigation activity zones) was $8.167 \pm$ $1.515(\mathrm{~N}=6)$. The resulting difference between the means $A$ and $B$ was $5.083 \pm 3.562$. Again, identification and quantification of Anopheles spp density using Gillies and de-Meillon protocol showed quite a high number of Anopheles spp densities in farrows followed by ponds and streams presenting the least Anopheles spp density. This should therefore bring to the attention of many that though urbanization indicates to some extent development, nevertheless could sometimes contribute to malarial transmission due to certain anthropogenic activities.
\end{abstract}

Keywords: Typhoid fever; Acute glomerulonephritis; Edema; Hematuria; Oliguria

\section{Introduction}

Changes in the environment as a result of natural as well as anthropogenic influences have led to many undesirable effect causing diseases and parasitic infections [1]. The outcome of some studies support the notion that urbanization is a ways to minimize malaria transmission due to reduction in breeding sites for the female Anopheles spp [2]. However, there are other activities such as the use of several sources of water for irrigation prevailing in these seemingly reduced malaria burden urban settings compared to the rural settings that promote the breeding of mosquitos and thereby transmitting malaria as well [3]. This makes it difficult to really come out clearly to agree fully with other studies indicating that urbanization promote the reduction of mosquito breeding leading to a drastic reduction in malaria transmission if real time effort are not taken to control the vector [4]. Despite repeated control measures to curtail the malaria transmission, this phenomenon is still on the increase to about $50 \%$ when there is conducive medium such as water availability for breeding of the vector [5]. Wastewater irrigation in many research findings have been revealed as a job creation opportunity for farmers, allowing them to take care of their household needs in terms of paying school fees, hospital and other servicing facility bills as well as meeting equally important ventures to enhance the family living condition [6]. These observed benefits are not different from farmers in Kumasi who as well will do everything possible to get access of water to irrigate their agricultural produce mainly vegetables [7]. Majority of irrigation farmers depend on water other systems of water for irrigation from storm drains mixed with domestic water from homes, hand dug wells, polluted streams and unprotected ponds to irrigate their agricultural produce [8] which are mostly unrestricted vegetables consumed by about $90 \%$ of the populace in the city [9]. The demand of vegetables in developing countries such as Ghana is increasing due to the education and awareness of its nutritive health implication which are mostly grown using wastewater [7]. However due to the demand, the supply must be there to meet the demand. The ponds and stagnant waters used by the farmers to irrigate their vegetables all year round are the habitats of these mosquitoes all year round. Although the mosquito larval stages require about 4-14 days for maturity, sometimes could be longer and can only be supported by an undisturbed water habitats. In the rainy seasons mosquito breeding is more pronounced due to the fact that more breeding habitats are created as in stagnant waters collected in ditches, trees, field containers and other suitable spaces available which mimic the water systems used for irrigation [8]. Effective and practically implementable measures must be put in place to reduce the malaria burden by taken into consideration all available means in which the vectors of malaria transmission could be reduce if not eliminated. In addition, other simple and cost effective irrigation technologies could be made known to farmers so that, the use of wastewater and other unhealthy means of irrigation could be avoided in other to reduce the burden of malaria on farm [10].

Major urban centres in Kumasi can boast of more farmers engaging in Agricultural (mostly vegetable) wastewater production which is patronized by many people within the communities. Though this could pose health problems, other research groups have focused on field assessment in terms of the vector.

This current study wanted to assess the situation on the field as well as real laboratory confirmed malaria case within the communities for policy makers to design policies based on clearly informed decisions. 


\section{Materials and Methods}

The study was conducted in the urban and periurban settings in the Kumasi Metropolitan Assembly. Prospective data from healthcare centres where analysed to score the cases of malaria within and without wastewater irrigated agricultural communities. In addition Anopheles larvae were identified and quantified in irrigation waters on the fields in the mornings from 006-10hrs from impoundments (furrow, ponds, streams and drains). Those that were not able to be identified were aseptically collected from the same time using standard sampling technique addressed by Robert et al. Sterile scooping container was dipped carefully and slowly into the water for mosquito larvae. After each dip, mosquito larvae in the sterile container were transferred into a sterile bottle. The bottles were covered with screwed caps and labelled to indicate place, type of water and sampling date. The samples were then quickly transported to the laboratory for breeding to adult stage for morphological identification of Anopheles spp [11].

\section{Adherence during sampling}

Dipping was always done slowly at the breeding sites approximately at an angle of $45^{\circ}$ to avoid disturbing the larvae. At least ten-minutes were allowed to elapse before another dip was carried out, to allow the larvae to return to the surface of the water. A maximum time of one day was allowed after heavy rains before collection of larvae water was made in order to avoid the likelihood of larvae been washed away after rains.

\section{Results}

Prospective malaria cases (7017) from health facilities within 77 communities in the Kumasi metropolis were assessed. The result revealed that, in general the communities where wastewater irrigation activities are paramount had higher prevalence of malaria cases compared with communities without wastewater irrigation activities (Figure 1).

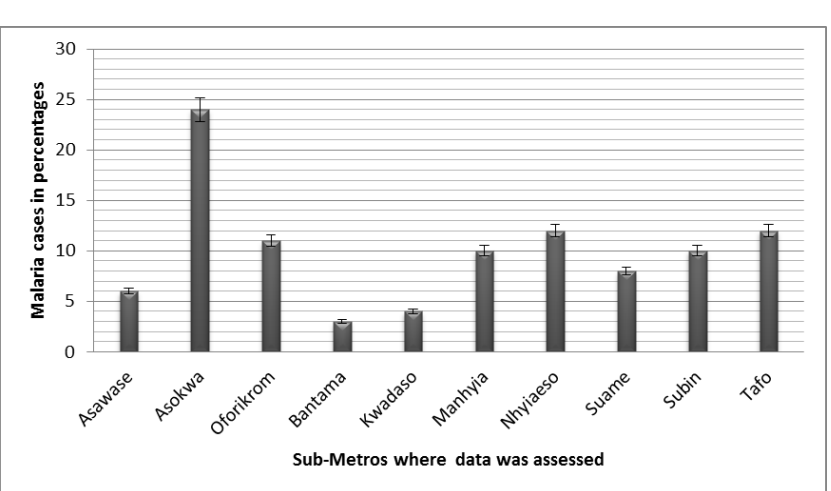

Figure 1: Malaria cases recorded within the Sub-Metros of KMA.

Additionally it was observed that, within the Kumasi metropolis, wastewater irrigation activities occur in about four (4) major submetros (Figure 1) with approximately 3698 reported malaria cases within 38 communities. Among the six (6) Sub-metros (Figure 1) where wastewater irrigation activities were seldomly practiced, 3319 malaria cases has been recorded. Comparing the result statistically (sub-metros with irrigation activities A verses sub-metros without irrigation activities $\mathrm{B})$ at $95 \%$ confidence interval $\mathrm{P}=0.1914$. The mean \pm SEM of $A$ is $13 \pm 3.816(\mathrm{~N}=4)$ and mean \pm SEM of $B$ is $8.167 \pm 1.515$ $(\mathrm{N}=6)$. The resulting difference between the means $\mathrm{A}$ and $\mathrm{B}$ is $5.083 \pm$ 3.562 .

Occurrences of Anopheles spp densities recorded within the sites where irrigation activities occur, Oforikrom Sub-metro presented the highest number of Anopheles spp density with Asawase Sub-metro showing the lowest Anopheles spp density. In the case of Nhyiaso* (Figure 2), there was a fairly large drainage system where all the wastewater irrigation farmers draw water with the help of pipe hoes to irrigate their vegetables but sampling of Anopheles density could not be achieved due to the nature of the drainage system.

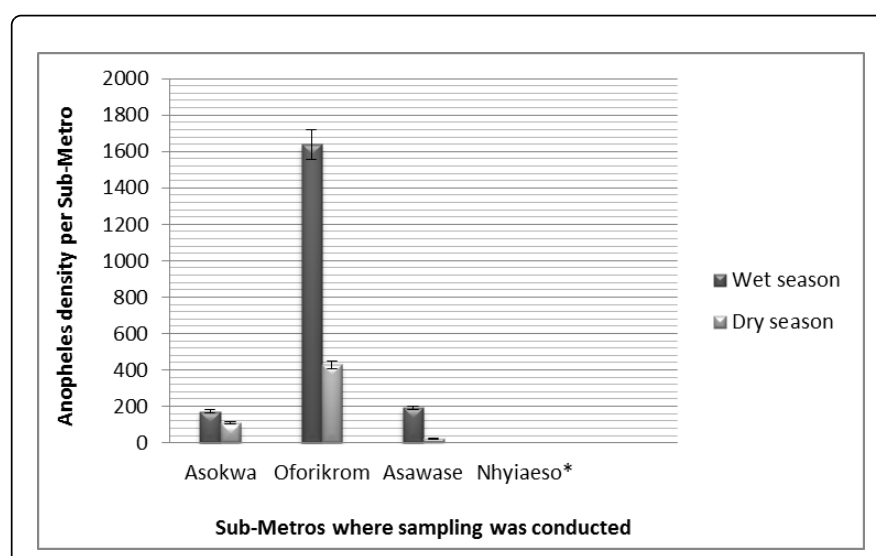

Figure 2: Anopheles spp density within irrigation sites per submetro in the dry and wet seasons.

Results presented by the study indicate some levels of differences in Anopheles spp densities of from ponds, streams and furrows (Figure 3) within the sites where irrigation activities were carried out. Results generally showed high numbers of Anopheles spp density in the wet season, which was usually twice that of the dry season. Furrow irrigated systems contained the highest Anopheles spp density both in the dry and the wet seasons (Figure 3). The stream which always had continuous flow had low Anopheles density.

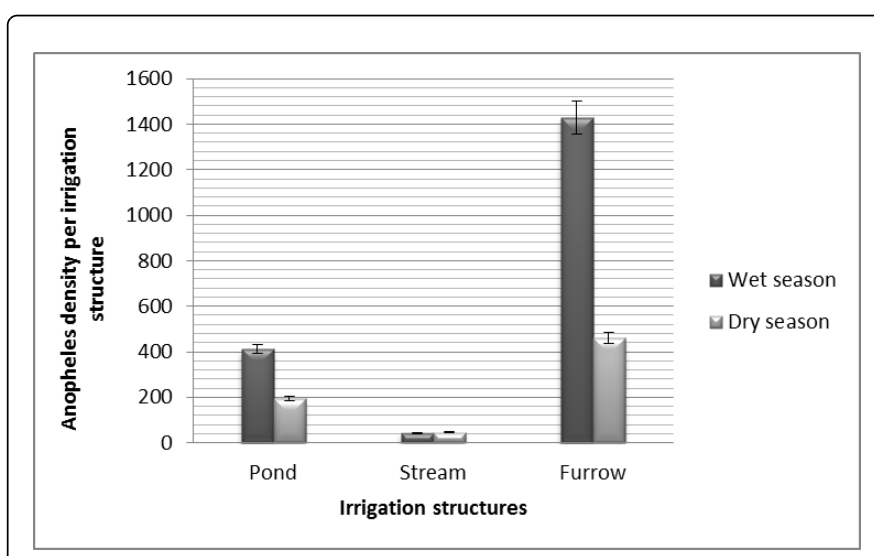

Figure 3: Anopheles spp density from different wastewater irrigation system. 


\section{Discussion}

There is an indication from this present study that communities with farmers engaging in wastewater irrigation activities present higher prevalence of malaria compared with communities without wastewater irrigation activities which have been similarly reported in a study by Smith et al. [12]. This is possibly due to the fact that the wastewater creates conducive environmental conditions for the breeding of mosquitoes [13] which subsequently transmit malaria and thereby increasing the increasing the malaria burden risk to vulnerable communities around these irrigation sites [14]. Apart from the wastewater, these farmers use poultry manure which are always heaped on the same site and during the rainy season especially, the used sacks, containers of weedicides and pesticides left unattended to collect water and increase the propagation of mosquitoes and transmit malaria [15]. Anopheles mosquito which is the main focus of this current study is believed to cause malaria which is the leading cause of illness in the Sub-Saharan Africa resulting in 8,200 cases daily and 3,000,000 illnesses every year [16]. Anopheles larvae has also been found to require about 4 days continuous in water to be able to hatch and develop into an adult mosquito ready to bite and possibly transmits malaria [15]. However, this study noted that farmers included in the study continuously irrigate their produce everyday which provides the opportunity for the mosquito larval stages to always hatch into adult stage without the eggs been damaged if all other conditions remain favourable as well. This study revealed during interaction sections with the farmers that, they always go to the hospital where they are diagnosed and treated with malaria. In addition, most farmers involved their family members in their farming activities and with their under-five (5) years children following them to the farms basically to play around, these children also suffer the burden of malaria [17]. This situation could easily arise due to the fact that, farmers and their household who constantly engaged in these irrigation activities go to their farms within the early hours of the day and stay till late in the evenings before retiring to their homes. However according to literature the biting periods of Anopheles mosquito are from dusk to dawn which is the period when farmers in this study continue to stay in their farms [15]. During the study, it was noted that farmers most of the times absent themselves as a result of ill health which may be due to malaria disease burden risk [18].

The study indicated quiet a high number of Anopheles density in the Oforikrom submetro compare to the rest of the submetros where irrigation activities similarly occur, this is because Oforikrom submetro has the majority of the wastewater irrigation farms. The outcome confirms the study conducted by Afrane et al. [19] which reported high numbers of mosquito densities in urban communities where agricultural irrigation practices occurred resulting in higher incidence of malaria cases within those areas. The farmers normally rely on any available water for irrigation; however the Oforikrom has a large storm drain and a river which runs through the communities which farmers used to irrigate their vegetables. This study noted that, the Asawase sub metro indicated the least mosquito density which was only recorded in the dry season with none recorded for the wet season which could be attributed to the fact that, in the Asawase sub metro, the availability of water for irrigation is minimal and this does not encourage a lot of farmers to cultivate vegetable through irrigation [20]. In the rainy season most of the farmers go back to their home towns in the Northern regions of Ghana to cultivate other crops by taking advantage of the rains.
With regards to the different irrigation water systems and sites, this study, however indicated that, throughout the year, the rainy or the wet season recorded high numbers of Anopheles density compared to the dry season which could perhaps be attributed to the fact that, in the wet season, the environment becomes humid and muggy which support the breeding of mosquitoes [21]. In addition in the rainy or the wet season pools of water can stand for long days which provide conducive habitat for mosquitoes to breed and develop to adult stage worthy of transmitting malaria.

Within the scope of this current study, mosquito larval densities at the irrigation sites were assessed from streams, ponds the as well as furrows (shallow pools of water created in between the vegetable beds). The comparative analysis indicated high Anopheles density in the furrows in both the dry and the wet seasons which could be attributed to the fact that, within these furrows are most stagnant waters located and with the sunlit couple with favourable temperatures breeding and maturity are enhanced [22]. Most of the furrows in this study were observed to be deepened by the farmers' footprints as they go about their normal irrigation activities around the edges of the vegetable beds which were noted to agree with the study by Afrane et al. [22]. The stream recorded the least Anopheles density with the reason been that, there is always a continuous flow of water from the stream which carried the eggs and the larvae away prematurely preventing natural breeding from taking place.

\section{Conclusion}

Areas dominated with wastewater irrigation activities in Kumasi had increase in malaria cases compared to areas without wastewater irrigation practices and the highest number of Anopheles spp density was recorded in the wet season compared to the dry season.

Policy makers and policy implementers should prioritised providing urban and peri urban agricultural farmers with simple and cost effective technologies for irrigation.

\section{Acknowledgment}

The authors acknowledge the contribution of the Norwegian Research Council, Norway and Department of Theoretical and Applied Biology, KNUST, Kumasi, Ghana.

\section{References}

1. Brunner FS, Eizaguirre C (2016) Can environmental change affected host/parasite-mediated speciation? Zoology 119: 384-394.

2. Hay SI, Guerra CA, Tatem AJ, Atkinson PM, Snow RW (2005) Urbanization, Malaria transmission and disease burden in Africa. Nat Rev Microbiology 3: 81-90.

3. Bonhee C (2016) Impact of Irrigation Extension on Malaria Transmission in Simret, Tigray, Ethiopia. Korean J Parasitol 54: 399-405.

4. Killeen GF, Fillinger U, Kiche I, Gouagna LC, Knols BG (2002) Eradication of Anopheles gambiae from Brazil: lessons for malaria control in Africa? Lancet Infect Dis 2: 618-627.

5. Alzahrani MH, McCall P, Hassan A, Omar AI, Abdoon AM (2017) Impact of Irrigation System on Malaria Transmission in Jazan Region, Saudi Arabia. Open J Trop Med 1: 7-15.

6. IWMI (2006) Recycling Realities: Managing Health Risks to Make Wastewater an Asset. Water Policy Briefing 17; IWMI and GWP, Colombo, Sri Lanka.

7. Obuobie E, Keraita B, Danso G, Amoah P, Cofie O, et al. (2006) Irrigated Urban Vegetable Production in Ghana: Characteristics, Benefits and Risks, IWMI-RUAF-CPWF, IWMI, Accra, Ghana. 
Citation: Abubakari A, Larbi JA, Abaidoo RC (2108) Implication of Urban and Peri-Urban Agricultural Wastewater Irrigation to Malaria Transmission in Kumasi. J Trop Dis 6: 275. doi:10.4172/2329-891X.1000275

Page 4 of 4

8. Asenso-Okyere K, Asante FA, Tarekegn J, Andam KS (2011) A review of the economic impact of malaria in agricultural development. Agr Econ 42: 293-304

9. Cofie OO, Drechsel P, Amoah P, Danso G, Gyiele L (2001) Improving rural-urban nutrient flows through urban and pen-urban agriculture. In: Rural- Urban Encounters: Managing the Environment of the Peri-urban Interface. London: DPU international Conference Paper.

10. Coene J (1993) Malaria in urban and rural Kinshasa: the entomological input. Med Vet Entomol 7: 127-137.

11. Gillies MT, de Meillon B (1968) The Anophelinae of Africa South of the Sahara (Ethiopian zoogeographical region). Johannesburg: The South African Institute for Medical Research.

12. Smith J, Nasr J, Ratta A (2001) Urban Agriculture: Food Jobs and Sustainable Cities, support by the United Nations Development Programme (UNDP).

13. Kinkenberg E, McCall PJ, Wilson MD, Amerasinghe FP, Donnelly MJ (2008) Impact of urban agriculture on malaria vectors in Accra, Ghana. Malar J 7: 151.

14. Smith T, Charlwood JD, Takken W, Tanner M, Speigelhalter DJ (1995) Mapping the densities of malaria vectors within a single village. Acta Trop 59: $1-18$.
15. Lawler SP, Lanzaro GC (2005) Managing mosquitoes on the farm. ANR Publication 8158.

16. National Malaria Control Programme annual report (2009) National Malaria Control Programme, annual report. Ghana Health Service.

17. WHO/UNICEF (2013). Progress on Sanitation and Drinking water.

18. Asante FA, Asenso-Okyere d' Almeida S, Mwabu G, Okorosobo T (2004) Economic burden of malaria in the African Region: Evidence from Ghana. Communicable Diseases Bulletin for the African Region 4: 1-3.

19. Afrane YA, Klinkinberg E, Drechsel P, Owusu-Daaku K, Garms R, et al. (2004) Does irrigated urban agriculture influence the transmission of malaria in the city of Kumasi, Ghana? Acta Tropica 89: 125-134.

20. Rosegrant MW, Cai X, Cline SA (2002) World water and food to 2025: Dealing with scarcity (International Food Policy Research Institute, Washington, DC).

21. Munhenga G, Brooke BD, Spillings B, Essop L, Hunt RH, et al. (2014) Field study site selection, species abundance and monthly distribution of anopheline mosquitoes in the northern Kruger National Park, South Africa. Malar J 13: 27.

22. Afrane YA, Lawson BW, Brenya R, Krupa T, Yan G (2012) The ecology of mosquitoes in an irrigated vegetable farms in Kumasi, Ghana; abundance productivity and survivorship. Parasites and Vectors 5: 233-239. 